\title{
Heavy Flavour Production at the Tevatron
}

\author{
Phillip Gutierrez* ${ }^{\dagger}$ \\ Department of Physics and Astronomy \\ University of Oklahoma, Norman, OK, USA \\ E-mail: ipgutierrez@ou.edu'
}

\begin{abstract}
We present results from the Fermilab Tevatron on $b$ and $t$ quark production in $p \bar{p}$ collisions at $\sqrt{s}=1.8 \mathrm{TeV}$. The $b \bar{b}$ cross section has been measured in both the central and forward regions. In addition, differential cross sections as a function of difference in azimuth and rapidity of the two $b$-quarks have been obtained. And the $t \bar{t}$ cross section in different channels, and limits on single-top production are also presented.
\end{abstract}

\section{Introduction}

Heavy quark production in $p \bar{p}$ collisions at the Fermilab Tevatron provides a quantitative means of testing perturbative QCD in the limit of large masses relative to $\Lambda_{\mathrm{QCD}}$. In hadronic collisions, heavy quark production at leading order (LO) occurs through both $q \bar{q}$ annihilation $(q \bar{q} \rightarrow Q \bar{Q})$, and $s$ and $t$-channel gluon-gluon fusion $(g g \rightarrow Q \bar{Q})$. The two processes dominate in different regions of phase space, with $q \bar{q}$ annihilation being the major process near the $Q \bar{Q}$ threshold and large $p_{T}$, and gluon processes dominant at low $p_{T}$. At the Tevatron, $b \bar{b}$ production is dominated by gluon processes, while $90 \%$ of the $t \bar{t}$ cross section is due $q \bar{q}$ annihilation. In addition to the LO terms, the next to leading order (NLO) terms for these reactions are now also available. These include gluon splitting, flavor excitation, gluon radiation, and loop corrections. The data discussed in this review are from $\approx 130 \mathrm{pb}^{-1}$ samples at $\sqrt{s}=1.8 \mathrm{TeV}$, collected independently by the CDF [i] and $\mathrm{D} \varnothing[\underline{2}]$ experiments.

\section{Production Results on Bottom}

The observed $b \bar{b}$ production cross section provides a direct comparison of data with perturbative QCD calculations. These calculations include the NLO terms [i]⿱一⿱㇒⿵冂⿰丨丨寸], which are comparable to the LO terms. The NLO QCD predictions show a large dependence on the renormal-

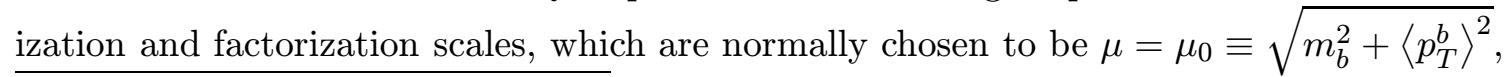

${ }^{*}$ Speaker.

${ }^{\dagger}$ on behalf of the D $\varnothing$ and CDF Collaborations. 
where $\left\langle p_{T}^{b}\right\rangle$ is the average $p_{T}$ of the $b$ and $\bar{b}$, and $m_{b}$ is the $b$-quark mass. In addition to the scale dependence, the predictions are sensitive to $m_{b}$, which is taken as $4.75 \mathrm{GeV}$. To estimate the uncertainty in the NLO QCD calculations, the scale is varied between $\mu_{0} / 2$ and $2 \mu_{0}$, while the $b$-quark mass is varied in the range 4.5 to $5.0 \mathrm{GeV}$.

The first measurements of hadronic $b \bar{b}$ production were carried out by the UA1 exper-

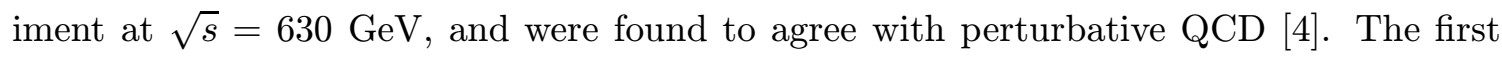
observation of discrepancy between calculations and data was reported at the Tevatron, where the $b \bar{b}$ cross section was found to be $\approx 2.5$ times larger than expected. Subsequent to these measurements, HERA [i5] results on photoproduction, and LEP [6] results on two-photon production of $b \bar{b}$, have found similar disagreements.

Several methods exist to extract $b$-jets from data. One way that CDF and D $\varnothing$ extract $b$-jets from data is by searching for jets with associated leptons. The jet is reconstructed using a cone algorithm, with a cone size of $\Delta R=\sqrt{\Delta \eta^{2}+\Delta \phi^{2}}=0.4$ for CDF, and $\Delta R=0.7$ for $\mathrm{D} \emptyset$. The jet is then tagged as coming from a $b$-quark if a lepton $(e$ or $\mu)$ is found within a cone $\Delta R^{\mu-\text { jet }} \lesssim 0.7$, where the cone size depends on the specific analysis. $\mathrm{CDF}$ also uses its silicon vertex tracker to identify tracks within a jet cone that come from secondary vertices.

Inclusive single $b$-quark cross sections have been reported previously. Here we concentrate on $b \bar{b}$ cross sections and correlations. These results, in addition to providing measurements similar to the single inclusive $b$-quark cross sections, provide information on the underlying QCD processes by exploring the kinematic relationship between the two $b$-quarks. CDF has measured the $\mu-b$ cross section, and used this to extract the $b \bar{b}$ cross section and the azimuthal correlation between the $b$ and $\bar{b}[\bar{i}]$. They select events that have a jet with $E_{T}>10 \mathrm{GeV}, \eta<1.5$ and tracks emanating from a secondary vertex. The muon used to tag the other $b$-quark, is required to have a $p_{T}>9 \mathrm{GeV},|\eta|<0.6$, and $\Delta R^{\mu \text {-jet }}>1$; the last requirement assures that the muon is not associated with the tagged jet. Figure shows the $\mu-b$ cross section, corrected for resolution, as a function of $p_{T}$ of the $b$-quark, along with a NLO QCD calculation [3in. The $p_{T}$ distribution of the $b$-quark is reconstructed using the properties of the $b$-jet and the $\mu$, the known detector resolution, and the model of Ref. 娣. The differential cross section shown in Fig $\underline{1}_{1}^{\overline{1}}$ is systematically larger than the

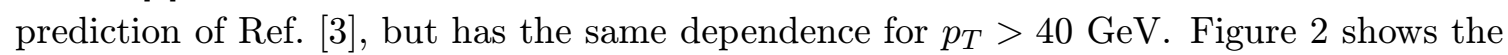
differential cross section for $\Delta \phi^{\mu \text {-jet}}$, which appears to differ systematically from the NLO QCD calculation. CDF has also measured the rapidity correlation between $b$-quarks produced in an event [i]. They require one $\mu$ tagged jet to be in the central region $(|\eta|<1.5)$. The events are then classified as central-central $(\mathrm{CC})$ or central-forward $(\mathrm{CF})$ depending the rapidity of the muon of the second $b$-quark; $|\eta|<0.6$ is central, and $2.0<|\eta|<2.6$ is forward. The ratio of the derived cross sections (CF/CC) is $R=0.361 \pm 0.033_{-0.031}^{+0.015}$, while NLO QCD yield $R=0.338_{-0.097}^{+0.014}$, providing good agreement.

$\mathrm{D} \varnothing$ has measured the $b \bar{b}$ cross section using the di-muon channel [ $[\overline{9}]$. The muons are required to have a $p_{T}>4 \mathrm{GeV}$ and $|\eta|<0.8$, and be associated with a jet $\left(\Delta R^{\mu-\text { jet }}<0.8\right.$ and $\left.E_{T}^{\text {jet }}>12.0 \mathrm{GeV}\right)$. The cross section was then calculated for the leading muon in the event. To extract the $p_{T}$ distribution of the $b$-quark from the muon $p_{T}$ distribution, the HVQJET Monte Carlo [1] $[1] \overline{0}]$ is used to determine the correlation between muon and 


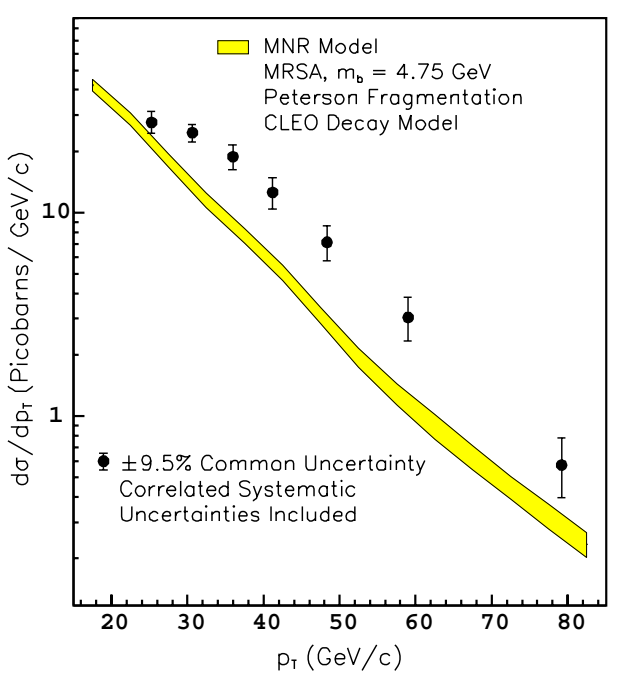

Figure 1: CDF $\mu-b$ differential cross section.

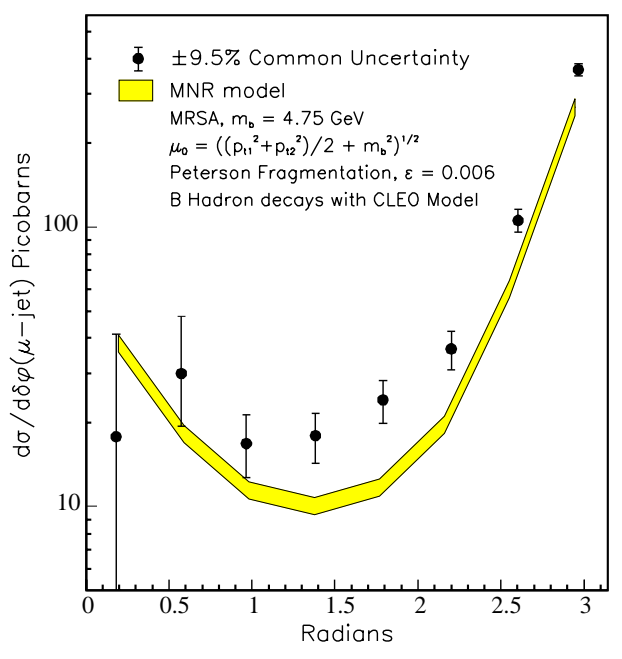

Figure 2: $\mathrm{CDF} \Delta \phi^{\mu-b}$ measurement.

$b$-quark. Figure to $\sigma\left(p_{T}^{b}>p_{T}^{\min }\right)$, and $p_{T}^{\min }$ is the $p_{T}$ of the $b$-quark for which $90 \%$ of the accepted events have a $p_{T}>p_{T}^{\min }$. As for the CDF measurement, $\mathrm{D} \varnothing$ observes a larger value of the cross section than expected from NLO QCD. The differential cross section for the difference in azimuth of the muons is shown in Fig. 19.9. Data and the NLO QCD prediction agree reasonably well. $\mathrm{D} \varnothing$ has also measured the $b$-quark cross section in the forward region, using single muons of $2.4<|\eta|<3.2$ and $p_{T}>2 \mathrm{GeV}$ [i]1] $\overline{1}_{1}$. The cross section is found to be a factor $\approx 4$ larger than the NLO QCD prediction.

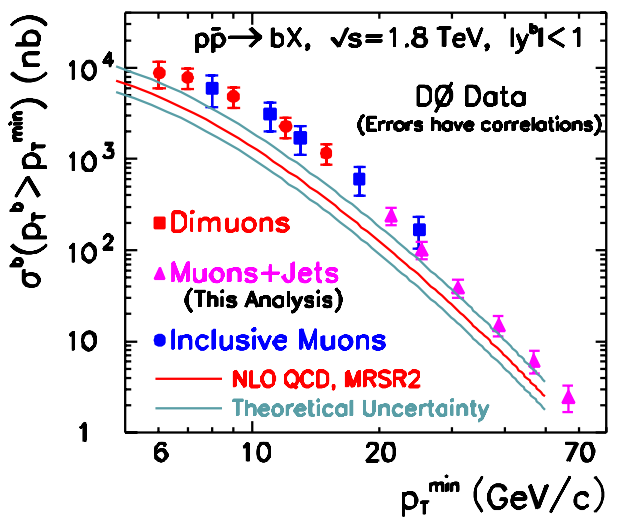

Figure 3: Summary of $D \varnothing$ cross section measurements in all channels.

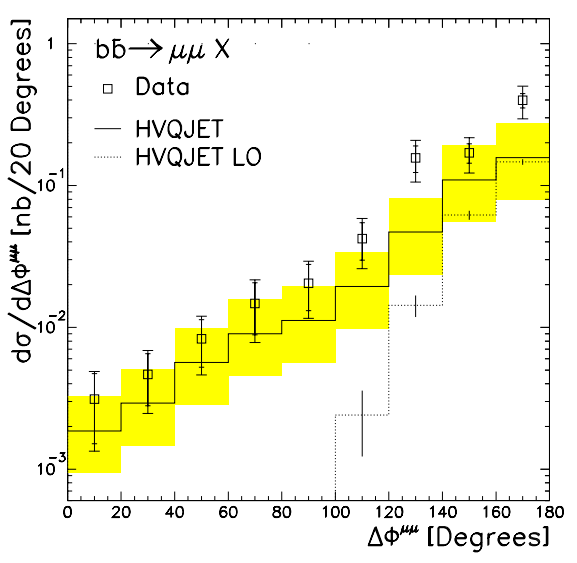

Figure 4: D $\varnothing \Delta \phi^{\mu-\mu}$ measurement.

The measurements described above are indirect, in that the observed kinematic variables are transformed to reflect the $p_{T}$ of the $b$-quark. This introduces a source of systematic error, because of ambiguities in fragmentation functions that are based on QCD gluon resummation calculations. On the other hand, the $E_{T}^{\text {jet }}$ distribution does not contain the 
troublesome collinear logarithms, because the jet $E_{T}$ does not depend on whether the $b$ quark carries all the energy. $\mathrm{D} \varnothing$ has measured the $b$-jet distribution for $E_{T}^{\mathrm{jet}}>25 \mathrm{GeV}$, for $\Delta R=0.7$ and $|\eta|<0.6$ and a muon tag [1] $\left.{ }_{1}^{1} \overline{2}\right]$. Figure for small values of $E_{T}^{\text {jet }}$ is systematically higher that the QCD calculation, but appears more consitent with QCD for large values of $E_{T}$.

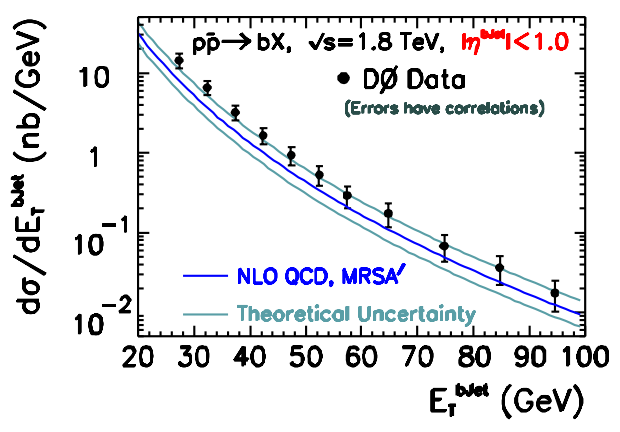

Figure 5: D $\varnothing b$-jet cross section.
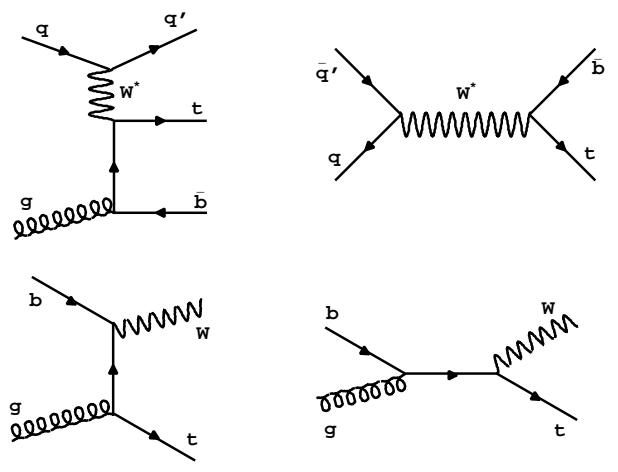

Figure 6: $\mathrm{LO} p \bar{p} \rightarrow t+X$.

\section{Production Results on Top}

After the discovery of the $t$-quark at the Tevatron [1] 1 inn to study its properties. These include improvements in the measurement of the mass, searches for standard model (SM) and non-SM decays, and production characteristics. The measurement of the $t \bar{t}$ cross section provides a goot test of perturbative QCD calculations in the limit of large mass scales.

To extract the $t \bar{t}$ signal from data, we use the characteristics of its dominant decay mode, $t \rightarrow W b$, which occurs $>99 \%$ of the time in the SM. The $W$ decays $1 / 3$ of the time to leptons $(e, \mu$, and $\tau)$, and $2 / 3$ of the time to 2 jets $(u \bar{d}$, and $c \bar{s})$. Given the difficulty in identifying $\tau$ leptons and the small number of $t \bar{t}$ events, the $\tau$ decay channel is ignored. Therefore, we search for events that have the following characteristics: $2 b$-jets +4 jets $(44 \%), 2 b$-jets +2 jets $+\ell+E_{T}(30 \%)$, and $2 b$-jets $+2 \ell+E_{T}(5 \%)$. As in the case of directly produced $b$-quarks, the $b$-jets in these events are tagged either through an associated lepton $(e$ or $\mu$ ), or through tracks within the jet that do not originate from the primary vertex. Using the all-jets mode, the single and di-lepton modes, CDF measured a combined cross section of $6.5_{-1.4}^{+1.7}$ pb [1] [1] be compared to the recent QCD calculation of Ref. [i] $\left.\overline{6}_{1}^{1}\right]$, which yields $5.8 \pm 0.4 \pm 0.1 \mathrm{pb}$.

At the Tevatron, the production of a single $t$-quark is possible through the weak interaction. The dominant processes are given in Fig. $\overline{6}_{1}^{\prime}$, and have expected SM cross sections of: $q g \rightarrow q^{\prime} t b, \sigma=1.47 \pm 0.22 \mathrm{pb} ; q \bar{q} \rightarrow t \bar{b}, \sigma=0.75 \pm 0.12 \mathrm{pb} ; b g \rightarrow t W, \sigma=0.15 \mathrm{pb}$; where $q g \rightarrow q^{\prime} t b$ and $q \bar{q} \rightarrow t \bar{b}$ are calculated at NLO, and $b g \rightarrow t W$ at LO [1] $\left.\overline{7}_{1}\right]$. Both CDF and $\mathrm{D} \varnothing$ concentrate on the $g q$ and $q \bar{q}$ processes, which are characterized by having 2 or 3 jets and a $W$, with two of the jets corresponding to $b$-quarks.

CDF requires the $W$ to decay through the electron or muon channel, with the lepton having $p_{T}>20 \mathrm{GeV}$ and $E_{T}>20 \mathrm{GeV}$ [i $\left.{ }^{1} \bar{g}_{1}\right]$. The event is required to have one, two, or 
three jets, with at least one being tagged as a $b$-jet through a displaced vertex. The mass of the lepton, neutrino, and the $b$-jet are required to be in the range 140 to $210 \mathrm{GeV}$, and a likelihood analysis is carried out using the scaler sum of the lepton, jet $E_{T}$, and $E_{T}$. CDF sets preliminary limits of $\sigma<13 \mathrm{pb}$ for $q g \rightarrow q^{\prime} t b, \sigma<18 \mathrm{pb}$ for $q \bar{q} \rightarrow t b$, and a combined limit of $\sigma<14 \mathrm{pb}$.

$\mathrm{D} \varnothing$ uses a neural network (NN) analysis to extract the signal [i] $[\overline{1} \overline{1}]$. The signal is broken up into four decay channels, $e+$ jets, $\mu+$ jets, with and without a $\mu$-tagged jet, and the two production channels, $q \bar{q}$ and $q g$. The background is divided into five samples, $W j j, W b \bar{b}$, $W W, t \bar{t}$, and misidentified leptons. One $\mathrm{NN}$ is trained for each signal and background, for a total of $20 \mathrm{NN}$. The $\mu$-tagged jets use the same NN as the untagged jets, except with different cutoffs on the output variable. The limits on single $t$-quark production for D $\varnothing$ are $\sigma<22 \mathrm{pb}$ for $q g \rightarrow q^{\prime} t b$, and $\sigma<17 \mathrm{pb}$ for $q \bar{q} \rightarrow t b$.

\section{Summary}

$\mathrm{CDF}$ and $\mathrm{D} \varnothing$ have measured the $b \bar{b}$ cross section along with correlations between the two $b$ quarks in an event. The observed cross sections are far larger than NLO QCD calculations predict, both in the central and forward regions. The correlation measurements on the other hand agree with NLO QCD, with the exception of the CDF $\Delta \phi^{\mu-b}$ measurement. The $t \bar{t}$ cross sections are in agreement with NLO QCD, and the upper limit on the single $t$-quark is consistent with expectation.

\section{References}

[1] F. Abe et al., Nucl. Instrum. Meth. A271, 387 (1988)

[2] S. Abachi et al., Nucl. Instrum. Meth. A338, 185 (1994)

[3] M. Mangano, P. Nason, G. Ridolfi, Nucl. Phys. B373, 295 (1992)

[4] C. Albajar et al., Phys. Lett. B256, 295 (1992); C. Albajar et al., Z. Phys. C61, 41 (1994)

[5] L. Gladilin Heavy flavour in photoproduction in these Proceedings

[6] V. Andreev Heavy flavour production in two-photon interactions in these Proceedings

[7] F. Abe et al., Phys. Rev. D53, 1051 (1996)

[8] F. Abe et al., Phys. Rev. Lett. D61, 032001 (2000)

[9] B. Abbott et al., Phys. Lett. B487, 264 (2000)

[10] M. Baarmand and F. Paige, HVQJET Monte Carlo Generator, private communication. Based on Ref. [3in.

[11] B. Abbott et al., Phys. Rev. Lett. 84, 5478 (2000)

[12] B. Abbott et al., Phys. Rev. Lett. 85, 5068 (2000)

[13] F. Abe et al., Phys. Rev. Lett. 74, 2626 (1995); S. Abachi et al., Phys. Rev. Lett. 74, 2632 (1995);

[14] T. Affolder et al., Phys. Rev. D64, 032002 (2001) This is the most recent result, see references within earlier results. 
[15] B. Abbott et al., Phys. Rev. Lett. 83, 1908 (1999) This is the most recent result, see references within earlier results.

[16] N. Kidonakis, E. Laenen, S. Moch, and R. Vogt, Phys. Rev. D64, 114001 (2001)

[17] M.C. Smith and S. Willenbrock, Phys. Rev. D56, 6696 (1996); T. Stelzer, Z. Sullivan, and S. Willenbrock, Phys. Rev. D56, 5919 (1997); A.P. Heinson, A.S. Belyaev, and E.E. Boos, Phys. Rev. D56, 3114 (1997)

[18] CDF collaboration, FERMILAB-CONF-00-290-E, (Nov 2000.) 5pp.

[19] V.M. Abazov et al., Phys. Lett. B517, 282 (2001) 